\title{
Philosophiques
}

\section{Paquet, L. Platon. La Médiation du Regard. Leyden, Brill, 1973,}

VI, 484 p.

\section{Georges Leroux}

Volume 2, numéro 2, octobre 1975

URI : https://id.erudit.org/iderudit/203033ar

DOI : https://doi.org/10.7202/203033ar

Aller au sommaire du numéro

Éditeur(s)

Société de philosophie du Québec

ISSN

0316-2923 (imprimé)

1492-1391 (numérique)

Découvrir la revue

Citer cet article

Leroux, G. (1975). Paquet, L. Platon. La Médiation du Regard. Leyden, Brill, 1973, VI, 484 p. Philosophiques, 2(2), 253-285. https://doi.org/10.7202/203033ar

Ce document est protégé par la loi sur le droit d'auteur. L'utilisation des services d’Érudit (y compris la reproduction) est assujettie à sa politique d'utilisation que vous pouvez consulter en ligne.

https://apropos.erudit.org/fr/usagers/politique-dutilisation/
Cet article est diffusé et préservé par Érudit.

Érudit est un consortium interuniversitaire sans but lucratif composé de l'Université de Montréal, l'Université Laval et l'Université du Québec à Montréal. Il a pour mission la promotion et la valorisation de la recherche. https://www.erudit.org/fr/ 


\title{
ÉTUDES CRITIQUES
}

PAQUET, L. Platon. La Médiation du Regard. Leyden, Brill, 1973, VI, 484 p.

\author{
par Georges Leroux
}

La philosophie de Platon se caractérise par la position des formes intelligibles. Cette thèse reçoit dans les dialogues des démonstrations diverses, tant par la variété des procédures utilisées pour légitimer l'existence d'un registre de réalité contraire au sens commun que par la pluralité des faits, des observations empiriques et des situations de discours d'où procédures et arguments sont repris. En ce qui concerne les procédures, Richard Robinson ${ }^{1}$ a clairement démontré qu'un double conflit embrouille la méthodologie platonicienne; il y a en effet une incohérence, dont il est difficile de disposer, entre l'épistémologie de Platon, marquée par la thèse d'un savoir catégoriquement certain, et la méthodologie de l'hypothèse. Celle-ci, d'autre part, entre en conflit avec l'usage fréquent de l'analogie et des images, à l'endroit desquelles, paradoxalement, Platon exprime souvent un jugement négatif. Cette deuxième incohérence est particulièrement notable dans les dialogues de la période centrale (Gorgias, Phédon, République etc...), où se rencontrent les grandes analogies platoniciennes: Ciré/âme, santé/justice, Soleil/Bien. De ces analogies, Platon paraît tirer plus qu'il n'a obtenu ailleurs de méthodes déductives, notamment en ce qui a trait à la distinction du sensible et de l'intelligible.

Quant aux faits, observations et discours dont proviennent les analogies et les arguments susceptibles de confirmer la thèse

1. Voir R. ROBINSON, Plato's earlier Dialectic. Oxford, 1953 (2e édition), en particulier le chapitre XII: «Analogy». 
des formes intelligibles, on a souvent noté, outre le fait qu'ils ne constituaient pas des démonstrations véritables, qu'ils n'étaient pas de nature exclusivement épistémologique. Le platonisme n'est pas construit uniquement sur la requête de fonder un savoir certain, stable, immobile. Les critères moraux, les normes esthétiques, les idéaux politiques, les nombres, associés à la thèse des formes, ont contribué à obscurcir un discours qui, s'il s'était limité à son versant épistémologique, aurait eu, sinon la plausibilité, du moins la netteté du réalisme. En effet, l'utilisation des notions de paradigme, de modèle dans des contextes autres qu'épistémologiques a contraint, au prix d'un forcement notoire, la thèse des formes à une interprétation en termes de «valeurs», qui a pour conséquence de gonfler le réalisme en un idéalisme. Celui-ci engendre autant de propositions qu'il se trouve de contextes pour lui en fournir le motif. La difficulté de faire coïncider chez Platon des propositions provenant de domaines aussi divers que l'art, la politique et les mathématiques ne laisse pas de solliciter les interprètes.

De ces difficultés relatives aux procédures et aux contextes de la philosophie platonicienne, L. Paquet a fait le fonds de son entreprise. Le principe déterminant de son étude est le suivant: une analogie est tout autant un système proportionné de rapports entre des termes que l'action et le mouvement reliant ces termes. En effet, par exemple, si la connaissance véritable est à l'intelligible ce que l'opinion est au sensible, cette formulation du système n'épuise pas la manière d'exploiter l'analogie. Elle cache même à vrai dire son support et son mouvement, qui sont présupposés, c'est-à-dire le sujet du rapport, son action. Cette première observation sur les présuppositions de l'analogie en appelle une seconde. Dans plusieurs cas, le système des rapports peut varier et subir des transformations sémantiques importantes sans altérer ce qui paraît être une constante de la philosophie platonicienne, la médiation du regard, action propre de l'analogie, attribuée à plusieurs sujers. Cette action peut être représentée comme le regard vers le paradigme ou le modèle, regard constitutif de l'analogie dans des systèmes ou domaines de «transposition » tels que la démiurgie, la mystique, etc... Du point de vue des questions que j'ai mentionnées au début, ce principe a des conséquences importantes qu'il importe d'énumérer avant de 
procéder à la lecture du livre de L. Paquet. Premièrement, de l'analogie le discours philosophique tire des propositions autant au niveau des termes ou entités mis en rapport que des «médiations» constitutives de ces rapports. En soi, cette conséquence est déjà une pièce de plus au dossier de l'analogie. Le rôle des médiations, pour obscur qu'il paraisse, peut en effet, si on parvient à le dégager suffisamment et à en montrer la constance, contribuer à aplanir les contradictions issues de la diversité des contextes de provenance des analogies et dont une lecture littérale fait l'embarras de l'interprétation. Dit autrement, dans les analogies constitutives du platonisme, il faudrait plutôt accentuer ce qui en elles est médiation et présupposition que ce qui en constitue les termes; plutôt le mouvement de la philosophie que sa structure ontologique. En second lieu, si ce point de départ est conduit jusqu'à ses conséquences méthodologiques, le conflit entre la méthode de l'hypothèse et la thèse du savoir certain s'amoindrit puisqu'elles n'ont pas la même portée et l'utilisation de l'analogie devient moins problématique, puisque les procédures analogiques ne sont pas véritablement heuristiques, mais tout simplement illustratives.

L'ouvrage de L. Paquet ne correspond guère par le style aux remarques générales que j'ai cru utile de formuler aux fins de le présenter correctement. Il ne s'agit ici nullement d'un essai d'allure spéculative. L'enquête qu'on nous présente est divisée en deux parties, très différentes d'inspiration et de méthode, mais toutes deux marquées plutôt par un souci philologique que par la discussion des méthodes du platonisme. Je présenterai ces deux parties dans l'ordre, en m'etforçant de souligner le lien des résultats philologiques aux questions sur l'analogie et la méthode que j'ai rappelées. Peu explicitées dans un livre surtout riche en inventaires de textes, ces questions en constituent néanmoins la direction. Je consacrerai ensuite une deuxième partie de cette étude à discuter brièvement la pertinence de ces questions sur l'analogie et des concepts qui servent dans le livre à les aborder. En troisième lieu, je voudrais soulever certains problèmes posés par les méthodes philologiques des analyses qui nous sont proposées. Ces méthodes ne peuvent éviter de ramener des questions d'ordre sémantique, qu'il est essentiel d'examiner pour faire 
évoluer maintenant la philologie par la sémantique, là où, à une période encore récente, une telle influence était encore inversée. ${ }^{2}$

La première partie de l'ouvrage est essentiellement une enquête philologique sur le verbe $\beta \lambda \dot{\epsilon}^{\prime} \pi \epsilon \iota \nu$. Cette enquête est jointe à deux chapitres sur l'artisan des temps homériques à Platon (Chap. I) et sur l'ouvrier, l'artisan et le démiurge chez Platon (Chap. II). Inspiré par un article classique de A. Diès sur la transposition des doctrines préplatoniciennes dans le platonisme, dont le sujet était surtout l'orphisme et la thétorique, L. Paquet veut d'abord effectuer dans cette perspective une analyse de la démiurgie. Le tésultat de ces deux premiers chapitres, qui débouchent sur l'importance du regard vers le «modèle» est d'entraîner les six chapitres suivants (III-VIII) qui constituent une sémantique du verbe $\beta \lambda \lambda^{\prime} \pi \epsilon t \nu$. Cette enquête littéraire doit venir corroborer les approches des premiers chapitres, en détaillant l'importance de la formule «porter son regard sur» pour l'interprétation de l'analogie du démiurge.

Le commencement de toute enquête philologique est Homère. L. Paquet lui consacre quelques développements, où sont repris les travaux de W. Theiler et de P. Chantraine. De la conception homérique, foncièrement positive, on passe à la dévalo-

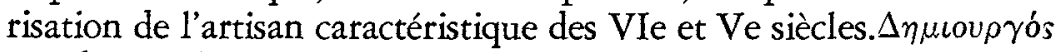
tombe en désuétude et pourra être repris par Platon pour nommer le Démiurge, alors que l'usage courant lui substitue les termes de $\beta a ́ \nu a v \sigma o s$ et de $\chi \epsilon \iota \rho \tilde{\omega} \nu a \xi$. L'un et l'autre véhiculent, en particulier chez les Tragiques, un mépris affiché du travail artisanal. Il est remarquable que la reviviscence du ônusovp ýs soit due à la tradition hippocratique et à la terminologie des opérateurs, du travail des «plasticiens». Le travail manuel est en effet d'abord, étymologiquement, chirurgie, $\chi \epsilon \iota \rho o v \rho \gamma i a$ (p. 37). Chez Platon, cette «chirurgie» sera fréquemment liée à l'emploi de $\beta$ ávavoos, comme, par exemple, dans Banquet

2. Il est difficile de séparer tout à fait le livre de L. PAQUET de celui de son maître Paul GRENET, Les arigines de l'analogie pbilosopbique dans les dialogues de Platon. Paris, Boivin, 1948. 
(203a6), Gorgias (450b9) et Politique (258d8 \& 259e11). C'est dans les Mémorables de Xénophon (I, 4, 2-9) que, à la suite de W. THEILER ${ }^{3}$, L. Paquet voit la première ré-utilisation, sans doute d'origine platonicienne, du démiurge dans un contexte cosmologique, c'est-à-dire comme artisan de l'Univers.

On trouve dans ce premier chapitre l'hésitation qui caractérise l'ensemble de l'étude de L. Paquet et sur laquelle je reviendrai plus loin ; l'approche qui se présente comme lexicale, c'est-àdire concentrée sur l'analyse de certains termes chez différents auteurs, donne lieu à des répertoires de textes très fouillés, mais cette approche hésite à se rigoriser dans le sens, selon moi souhaitable, d'une analyse sémantique pour toujours se disperser dans des considérations sur l'analogie.

Le second chapitre est consacré au démiurge platonicien. L. Paquet s'applique d'abord à discuter l'attitude paradoxale de Platon à l'endroit de la démiurgie: le mépris de l'artisan et des classes inférieures cohabite chez lui avec une utilisation analogique appuyée des métaphores techniques. Les contextes où se

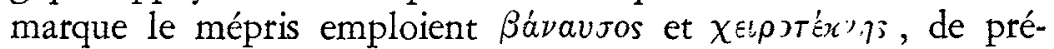
férence à $\delta \eta \mu$ lov $\rho$ ós (p. 46). Ce terme paraît réservé à des spécialistes, connaisseurs de leur art et estimés par Platon à proportion du savoir requis pour l'exercice de leur $\tau$ té $\chi \nu \eta$. Dès lors, le sens profond de la démiurgie, c'est l'action finalisée, postulat fondamental du platonisme, dont le sujet peut être le nomothète, le Démiurge créateur, et tous les autres rôles associés à la production (p. 52). La transposition du contenu préplatonicien atteint son achèvement dans le cas de l'attribution des prédicats de la démiurgie à la divinité. C'est ainsi que L. Paquet interprète Cratyle (425d5), République (415a, 507c, 596c5, $597 \mathrm{b6}$ ), puis dans la foulée de ces textes, ceux plus problémati-

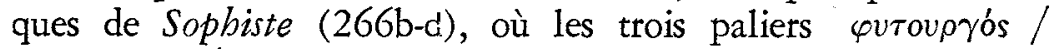

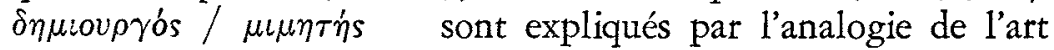
humain et de l'art divin. Dans ce processus de transposition de la figure du démiurge, les textes du Timée représentent la limite de la sublimation (p. 62). La production finalisée a cessé d'être le thème dominant de la transposition; elle laisse la place à

3. Cf. W. THEILER, Art. «Demiurgos», Reallexikon für Antike und Cbristentum, col. 696. 
l'abstraction des concepts d'intelligence et d'art, dont le monde se révèle pénétré de par sa fabrication divine. Ce chapitre se termine par la mention des textes des Livres X et XII des Lois, où les concepts du Timée se dépassent dans une véritable théodicée.

Je reviendrai plus loin sur le sens qu'il y a à parler de l'analogie du Démiurge, dont je serais porté à faire valoir le caractère fonctionnel et philosophique plutôt que littéraire et analogique. La lecture de Paquet me semble trop marquée par une interprétation littéraire de la tradition de la figure démiurgique, alors que cette histoire littéraire ne prend son sens que si on la reporte sur l'histoire réelle, socio-économique, de la démiurgie ancienne et en général des fonctions. ${ }^{4}$ Ce report est, en effet, la seule garantie d'objectivité dans l'interprétation de textes qu'il est risqué de faire giisser trop rapidement vers une interprétation de type analogique, guidée par une théodicée postérieure. Celle-ci n'est toujours que le résultat déformé de la philosophie platonicienne, à travers des siècles d'exégèse chrétienne, plutôt que son sens ou sa règle d'interprétation. Je ne m'arrêterai pas cependant à critiquer les interprétations de détail de ce chapitre, qui n'est dans l'esprit de l'auteur que le préliminaire de son sujet. De ce démiurge, en effet, L. Paquet veut d'abord retenir le regard.

Les chapitres III à VIII forment à eux seuls une recherche autonome sur le verbe $\beta \backslash \dot{\epsilon} \pi \epsilon i \nu$. Ce travail minutieux est un inventaire de tous les textes antérieurs à Platon où figure ce verbe. Il est important de noter ici que L. Paquet tient à présenter cette recherche comme une recherche sur une image (p. 70). C'est d'abord la forme verbale simple qui fait l'objet de l'analyse (Chap. III), avec ses nuances de regard témoin, de spectacle douloureux, de voyance, d'examen et de considération.

4. Pour le complément historique voir K. MURAKAWA, «Demiurgos》, Historia VII (1957) 383-415; pour une interprétation fonctionnelle, voir Luc BRISSON, Le même et l'autre dans la structure ontologique du Timée de Platon. Un commentaire systématique du Timée de Platon. Paris, Klincksieck, 1974; Chapitre I, pp. 27-106. 


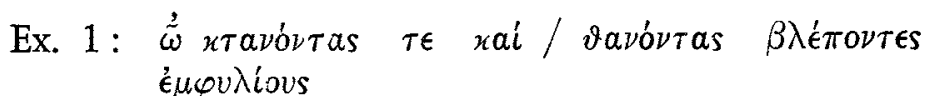

SOPHOCLE Antigone, 1263-64

Ah! la raison qui déraisonne, erreurs obstinées semeuse de mort, vous le savez du moins, vous qui voyez ici des meurtriers, des morts issus d'un même sang! [Trad. P. Mazon]

$\grave{A}$ ces multiples significations du regard physique, il faut ajouter la signification intellectuelle. $\mathrm{L}$. Paquet cite le texte suivant, où il reconnaît un regard non-physique, mais aussi des «attaches sensibles» (p. 82):

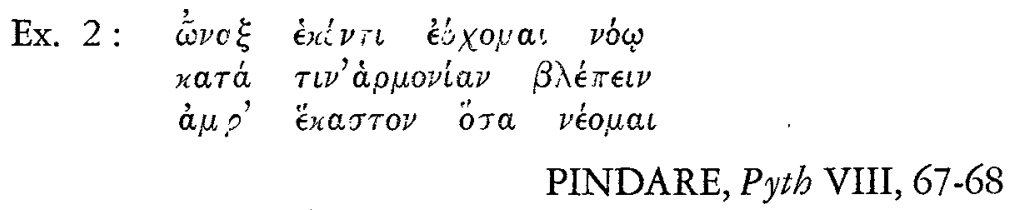

Ô Souverain je souhaite que tu jettes un regard favorable sur tout ce qu'en tout temps je chante sur des modes divers.

[Trad. A. PUECH]

Plusieurs exemples extraits des Tragiques sont témoins d'une évolution vers une signification abstraite. Ce sont ensuite des locutions telles que «voir le jour» et diverses utilisations du verbe avec des accusatifs de qualité. Le chapitre IV analyse les

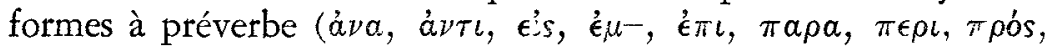
$\dot{v} \pi 0)$; de celles-ci, $\pi \rho \circ \sigma \beta \lambda \dot{\lambda} \pi \epsilon_{\imath} \nu$ est la forme le plus généralisée et son objet est invariablement un être vivant. L'emploi du verbe avec préposition fait l'objet des chapitres V et VI ( $\beta \lambda \dot{\epsilon} \pi \epsilon \iota \nu$ eis, $\beta \lambda \epsilon^{\prime} \epsilon \nu \pi \rho$ ós ) ; comme dans les chapitres précédents, ce sont surtout les textes des Tragiques qui sont mis à contribution, où se donne à voir clairement l'alliance du regard et du jugement. Par rapport à ces emplois, les textes de Xénophon constituent une exception (p. 127), alors que ceux des orateurs accentuent l'intellectualisation du «regard». Une remarque sur Aristote (p. 132-33) confirme la tendance de cette évolution: on ne 
trouve chez lui aucune trace d'un emploi de $\beta \lambda \epsilon ́ \pi \epsilon \iota \nu$ au sens de regard physique. L'insertion de la préposition $\pi \rho$ ós introduit, par la direction qu'elle impose au regard, la notion de distance et de prestige : regard vers un être supérieur.

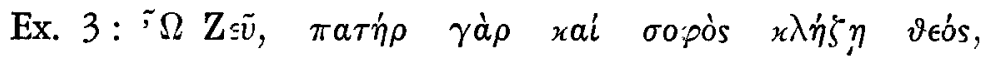

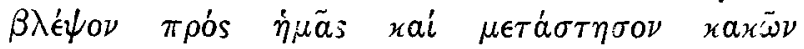

EURIPIDE, Hélène, 1441

Ô Zeus, Dieu paternel et sage tout ensemble, abaisse tes reg.zrds vers nous, délivre-nous de nos

maux.

[Trad. L. Méridier]

Ce thème est de tous le plus fréquent (p. 150) et il se prolonge dans celui de l'enquête, c'est-à-dire du regard porté vers l'inconnu prestigieux et attirant, mais aussi dans la direction de l'objet de valeur, du critère, du modèle normatif.

$\grave{A}$ ces résultats, les deux chapitres suivants (VII et VIII),

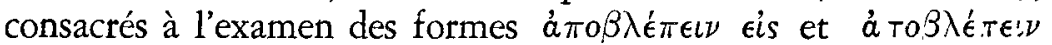
$\pi \rho o_{j}$ apporteront une confirmation détaillée. Ce dernier verbe en effet réfère rarement à la seule vision corporelle et sa signification paraît déterminée par l'objet visé, en général une valeur importante, vénérable (tombeaux des pères, actions héroïques etc...). Dans la panoplie des textes invoqués, Aristote et Xénophon demeurent des exceptions, autant pour la forme simple que pour les formes à préverbe et avec prépositions. Ce fait important, rapidement évacué par l'auteur, doit retenir notre attention. Nous y reviendrons plus loin.

Héritier de cette longue tradition littéraire, Platon n'a pu éviter d'en incorporer les nuances. La deuxième partie de l'ouvrage (IX-XIV) est composée de six chapitres très différents par l'objet et la méthode; tous ont néanmoins pour but de faire voir la manière dont le discours platonicien transpose l'image et l'analogie du regard et intègre les significations du verbe $\beta \lambda \epsilon \epsilon \epsilon \iota \nu$. L'enquête littéraire laisse ici la place à une analyse stylistique (IX), à des remarques d'ordre sémantique (X), puis à des discussions des contextes de la transposition philosophique: la visée de l'artisan (XI), la vision de l'initié (XII) et la vision de l'ar- 
tiste (XIII). Un chapitre final fait le point sur cette médiation du regard, thème commun des analyses précédentes (XIV).

De tous ces chapitres, le chapitre IX (une formule de récurrence) m'a paru le plus riche, bien que souvent susceptible d'objections. L'inspirateur ici n'est plus A. Diès, mais E. Desplaces ${ }^{5}$, qui avait remarqué comment, dans le texte de Lois XII, 962-965

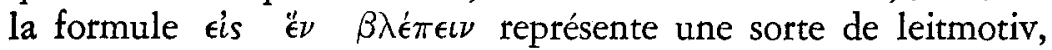
de thème dominant, selon l'expression de $\mathrm{H}$. Cherniss ${ }^{6}$. Mettant à profit les travaux de René Schaerer et ceux plus discutables de V. Goldschmidt ${ }^{7}$, L. Paquet tente de reporter sur la structure formelle des dialogues un schème stylistique d'emploi des verbes du regard. Schaerer, de façon tempérée, et Goldschmidt, de manière plus triomphante, ont en effet dégagé un modèle de la progression dialectique des dialogues, marqué par des temps forts et des articulations repérables dans le texte. Il est du plus haut intérêt d'apprécier de ce point de vue le résultat de la recherche de L. Paquet, intéressé à retrouver des marques textuelles de ce modèle. Cette contribution pourra se joindre aux travaux de H. Thesleff ${ }^{8}$ sur les marqueurs stylistiques chez Platon. L. Paquet soutient que la récurrence des verbes à l'étude se caractérise par leur insertion dans les passages charnières du dialogue platonicien, où l'enquête empirique et linguistique tourne à l'aporie et où le discours est contraint de «se tourner» vers l'Idée, vers l'Essence. En Ménon (96d5-el), dialogue caractérisé par une articulation particulièrement nette, le verbe $\beta \lambda \epsilon ́ \pi \epsilon \iota \nu$ marque la

5. E. DESPLACES, Une formule platonicienne de récurrence. Paris, 1929. Voir pp. 3-4.

6. In Gnomon (1953) 376, n. 3.

7. Cf. René SCHAERER, La question platonicienne. Étude sur les rapports de la pensée et de l'expression dans les Dialogues. Deuxième édition revue et augmentée d'une postface. Paris, Vtin, 1969. Ce livre est présenté dans la bibliographie de CHERNISS comme le meilleur sur l'écriture de Platon. Cf. «Lustrum》, Internationale Forscbungsberichte aus dem Bereich des klassischen Altertums 4 (1959) 228. L. Paquet suit plutôt V. GOLDSCHMIDT, Les Dialogues de Platon. Structure et méthode dialectique, Paris, PUF, 1963 (2e édition). Voir le compte rendu de R. Robinson, dans Mind 58 (1949) 246-49.

8. THESLEFF, H., Studies in the style of Plato. Helsinki, 1967 (Acta pbilosophica fennica, Fasc. XX). Ce livre constitue une recherche rigoureuse et originale, sans exemple dans l'érudition platonicienne. On ne peut en attendre ni une théorie brillante sur la dialectique, ni des schémas généraux, mais on y trouvera des analyses stylistiques précises et quelques remarques suggestives. 
reprise du discours valide, bien orienté. Il en est de même dans Polit. (264a8-b6). Ailleurs par exemple dans Banquet (198a4), le regard vers Eryximaque indique le centre de l'œuvre, tout comme en Phédon (117b). Dans le Parménide, (130e-135b), la construction se révèle être un enchâssement signalé par les for-

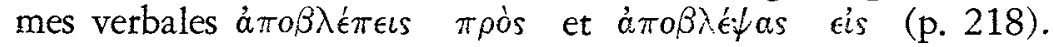
Il y a là donc comme une ponctuation du texte, tout à fait remarquable, et il est regrettable que cette partie du chapitre n'ait pas donné lieu à une démonstration plus étendue, c'est-à-dire indépendante du modèle de Goldschmidt dont la validité me paraît très problématique. L'appel de l'Essence, le moment définitionnel et tous ces concepts interprétatifs se révèlent assez vides quand il s'agit de passer à une analyse rigoureuse des marques dans les textes. Malgré ses limites, le travail de Thesleff paraît plus assuré de ses procédures.

Les développements suivants de ce chapitre laissent ce sujet pour se concentrer sur les objets compléments du verbe, dans le parcours des dialogues. Moins soucieux d'une analyse stylistique détaillée, ils sont à proportion moins riches de résultats. On y voit que $\beta \lambda \epsilon_{i} \pi \epsilon \nu$ commande des objets de valeur, tels la vertu, les nombres, la philosophie ou encore l'objet même du dialogue, comme par exemple, la Justice dans la République, l'argument important et enfin l'Essence, l'Idée. Les textes de la République, en particulier l'allégorie du livre VII, donnent lieu à des analyses de l'analogie du regard, de la lumière et de la vision où l'accent est mis sur la place qu'y tient le verbe $\beta \lambda \epsilon \in \epsilon l \nu$, en comparaison avec les verbes $\dot{o}_{\hat{\kappa}} \tilde{a} y^{\prime}, \quad \varkappa a \vartheta \vartheta_{0} \tilde{a} \nu$ et $\vartheta \varepsilon \tilde{a} \tau \vartheta q_{l}$. (p. 243) Par rapport à ces derniers, il ne comporte aucune nuance concrète; le verbe $\beta \lambda \epsilon \in \epsilon \iota \nu$ est tout entier absorbé par le haut, par la conversion à l'intelligible. Les trois exemples suivants illustrent cette thèse; il est intéressant de noter que dans l'exemple 4, deux verbes figurent avec des compléments de registre différent.

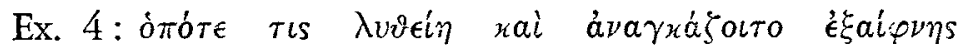

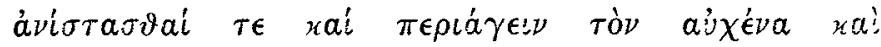

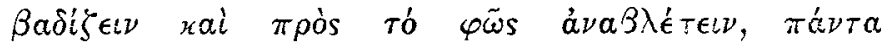

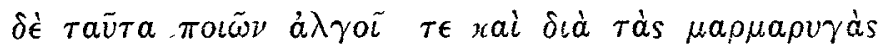

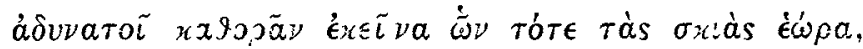


[La caverne] Qu'on détache un de ces prisonniers, qu'on le

force à se dresser soudain, à tourner le cou, à marcher, à lever les yeux vers la lumière, tous ces mouvements le

feront souffrir, et l'éblouissement l'empêchera de regarder

les objets dont il voyait les ombres tout à l'heure.

[Trad. E. Chambry]

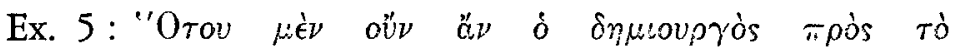

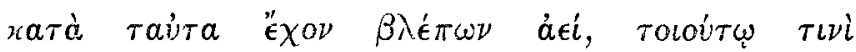

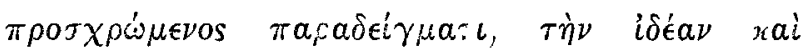

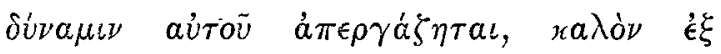

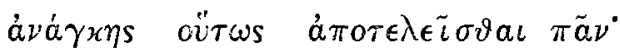

Timée 28a6-9

Toutes les fois donc que l'ouvrier, les yeux sans cesse fixés

sur ce qui est identique, se sert d'un tel modèle, toutes. les

fois qu'il s'efforce d'en réaliser dans son ouvre la forme

et les propriétés, tout ce qu'il produit de cette façon est nécessairement beau.

[Trad. A. Rivaud]

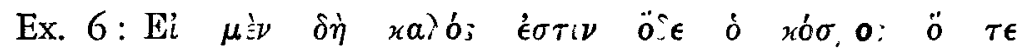

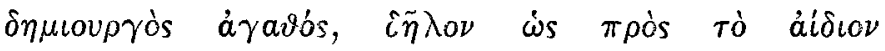
$" \epsilon \lambda \epsilon \pi \epsilon \nu-$

Timée 29a2-4

Or si ce monde est beau et si l'ouvrier est bon, il est clair

qu'il fixe son regard sur le modèle éternel.

[Trad. A. Rivaud] 


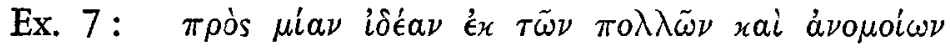

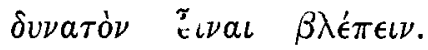

Lois, XII, 965c1-3

[Y aurait-il donc, pour qui que ce soit et à propos de quoi

que ce soit, une méthode plus exacte de recherche et d'observation, que de savoir] porter son regard de la diversité

du multiple sur l'unité de la forme?

[Trad. A. Diès]

Ces exemples diffèrent sensiblement de l'entrée lexicographique de $\beta \lambda \epsilon \in \pi \epsilon \nu$ dans Liddell, Scott \& Jones et ce problème relève de la méthode de $L$. Paquet, dont je discuterai plus avant. Comment se présente l'ensemble des emplois platoniciens quand on les compare aux emplois de ses prédécesseurs (p. 250ss)? La fréquence en est d'abord keaucoup plus grande; ensuite, Platon utilise surtout les formes avec préposition ( $\pi \rho$ ós ou $\epsilon \dot{i}$ ) et la

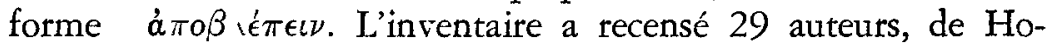
mère à la période hellénistique ; Platon emploie 89 fois $\beta \lambda \epsilon \in \pi \epsilon l \nu$ $\pi$ cós contre 23 fois chez Aristote et 39 fois dans les autres textes. Un intéressant tableau fournit d'autres chiffres (p. 250, note 3), et il faut déplorer que L. Paquet ne les exploite pas. Il se concentre plutôt sur l'importance de l'analogie du regard, fixé dans la direction d'une valeur. Cette analogie va de pair, au niveau de l'écriture, avec une transposition lexicale: les significations concrètes du verbe sont disparues chez Platon, où tout est regard de l'esprit, prise en considération, référence, attente. Cela tient selon L. Paquet aux contextes de la double image de l'artiste et de l'initié qui, comme celle du démiurge, est l'objet d'une transposition idéalisante dans le platonisme.

Je reviendrai, dans ine partie plus critique, sur les problèmes posés par ce type d'analyse lexicale, construite selon moi à partir d'une distinction des «significations » qui risque d'égarer. Il me paraît important cependant de noter immédiatement que les exceptions dans ce jartage des significations, c'est-à-dire Aristote et Xénophon, représentent comme Platon des auteurs de textes philosophiques. Si donc la notion d'une évolution de 
significations plus matérielles vers des significations plus abstraites recouvre en gros l'histoire littéraire qui conduit au discours philosophique, on ne peut attribuer au seul Platon le génie de la transposition du regard. Par ailleurs, des significations multiples cohabitent chez les uns et chez les autres. L'explication, linguistique et simple, de cet état de choses réside dans le fait que la signification est toujours tributaire de contextes et n'existe pas à l'état lexical séparé, c'est-à-dire attachée à des formes lexicales hors discours. Je m'en voudrais d'insister davantage sur ce point.

Plus intéressante est la notion de transposition, lorsqu'elle concerne tout un ensemble, tout un système de rapports analogiques entre deux discours et non plus seulement une seule forme verbale. Faute d'avoir toujours distingué ces deux aspects, le travail de L. Paquet rencontre des obstacles sérieux. À partir du chapitre X, l'analyse lexicale est laissée de côté au profit de considérations sur le contenu philosophique des images et des contextes. À partir d'un texte, tel que Alcibiade (133 a-c), l'auteur s'efforce d'abord de montrer la polyvalence du regard qui est à la fois vision et visée (Chap. X), contemplation et orientation. Regard porté sur la forme unique à partir de la diversité du multiple (Lois 965c2 ; voir ex. 7), $\beta \lambda \epsilon \epsilon \epsilon \iota \nu$ porte à la fois le but de l'activité de l'art ét la vision synoptique, terme de l'ascension mystique. Dans le discours philosophique, le terme paraît donc retenir des significations attachées à ses contextes d'origine; en vérité, plus que le terme, ce sont ces contextes eux-mêmes qui sont en totalité transposés, comme s'appliquent à le montrer les chapitres suivants. Les deux méthodes sont donc ici fusionnées.

Le premier de ces contextes est le plus connu; c'est celui de la Démiurgie, auquel L. Paquet a déjà consacré un chapitre. Reprenant (Chap. XI) l'interprétation dans le sens plus précis du lien qui unit l'activité du démiurge à son regard, L. Paquet isole dans les traits de l'analogie du démiurge ceux de la finalité de la production, dans sa double dimension de $\tau \dot{\epsilon} x \nu \eta$ et de qualité. Comme l'artisan, l'homme de bien, (homme d'État idéal, nomothète, etc...) vise l'excellence ( $\mathfrak{a}_{0}, \epsilon_{\tau} \dot{\eta}$ ), et c'est sur le modèle de l'intelligence technique et démiurgique que le «poli- 
tique » doit exercer sa «visée ». Dans les textes des Lois, qui dépassent sur ce point ceux de la République, cette visée est

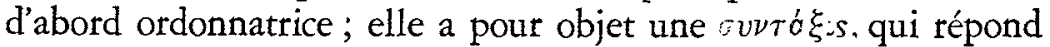
bien au contexte cosmologique où s'opère depuis le Timée la transposition de la démiurgie. Cette visée cosmologique accueille à la fois le souci platonicien de la rationalité et son expression dans les termes de la visée technique du démiurge.

Il s'en faut cependant que l'utilisation de l'analogie du démiurge dans le sens de la visée soit constituée comme une philosophie de la raison. C'est l'Introduction du Modèle, c'est-àdire de la forme, qui distingue le discours philosophique. Dans un chapitre remarquablement articulé (XII : La vision de l'Initié), L. Paquet reprend le travail de A. Diès et de Paul Grenet, et s'applique à démontrer comment Platon charge "d'un contenu proprement philosophique les valeurs latentes de l'orphisme et des. Mystères ». Ce sujet rebattu était difficile à aborder. Le mérite du travail de L. Paquet est ici de procéder synthétiquement. Il fait d'abord voir comment Platon rejette une religiosité excessive, faite de charlatanerie et de pratiques à tendance théurgique, pour n'accepter qu'une mystique purificatrice. Je crois que Paquet a tort de minimiser autant qu'il le fait l'importance du corybantisme et du bachisme chez Platon ${ }^{9}$, et je pense qu'en général il adopte une attitude rationaliste où perce un mépris irritant des religions antiques; cette attitude me paraît impropre autant à l'histoire de la philosophie qu'à l'histoire des religions. On se trouve en effet ici devant le paradoxe suscité par toute interprétation d'une philosophie en termes de transposition à partir d'autres sphères du discours. Le présupposé est celui de la purification. Je m'attarderai à discuter ce problème plus loin, mais il me paraît important d'en signaler l'incidence en faisant cet exposé du livre. L. Paquet n'estime guère les contenus transposés dans le platonisme; il n'est pas du tout assuré que Platon en ait jugé ainsi (p. 298). La divination, le délire, le têve sont des composants du platonisme qui ne sont pas seulement transposés, ils sont constituants; il n'v a pas d'au-delà ou de revers de l'irra-

9. Cf. I.M. LINFORTH, "The corybantic rites in Plato». University of California Publications in Classical Pbilology 13, 5 (1946) 121-162. On trouvera dans ce livre une attitude plus ouverte. 
tionnel chez Platon ${ }^{10}$. La philosophie n'est pas comme la version rationnelle de ces discours. Te citerai ici un seul texte, qui confirme une interprétation, - plutôt traditionnelle d'ailleurs, - du Banquet et du Phèdre; il s'agit du chour des vieillards dans le livre II des Lois, qui, bien qu'étant le chœur de la raison, est néanmoins le chour de Dionysos ${ }^{11}$. Je ne crois donc pas que le texte de l'Ion sur la poésie soit une satire qui emprunte le détour de l'image bachique pour tourner en dérision l'exaltation poétique; ce serait priver le Phèdre de tout son sens que de suivre ici L. Paquet (p. 316). Je ne crois pas non plus que Platon ait considéré comme une pathologie (p. 318) le dionysisme ou lorphisme. On s'en tire vraiment un peu facilement en parlant d'un paradoxe de plus à mettre au compte de «ce génie si complexe» (p. 319).

Cette remarque critique, prématurée, m'a paru nécessaire au moment d'exposer cette partie du livre où l'auteur montre comment Platon, malgré l'attitude réservée sinon méprisante qu'il entretient à l'endroit des artisans, des mystères et des artistes, va néanmoins couler dans ce moule ses intuitions les plus chères (p. 320). La divination, pour prendre un premier exemple, constitue pour L. Paquet un tel moule pour l'idée du commerce avec le divin et fondamentalement pour la communication avec le monde intelligible. Les Mystères sont un second exemple. Contrairement à L. Paquet, je crois ces exemples constitutifs du platonisme; ce ne sont pas des conques vides où le bernardl'ermite de l'idée vient faire son nid. On peut en donner une démonstration a priori: sans la divination, sans les Mystères, la mystique platonicienne s'évanouit puisqu'elle ne les transcendè pas. Il faut toujours garder en vue que la République s'ouvre et

10. Cf. I.M. LINFORTH, «Telestic Madness in Plato». University of California Publications in Classical Pbilology 13 (1946) 163-172. Et aussi, bien entendu, le livre de E.R. DODDS, Les Grecs et l'irrationnel, Paris, Aubier, 1965. Trad. Gibson (1959). On trouvera dans le recueil Divination et Rationalité, édité par Jean-Pierre Vernant (Paris, Seuil, 1974), un article de Luc Brisson, «Du bon usage du dérèglement», pp. 220-248, qui aborde ces questions, surtout dans le Timée.

11. Ce texte sur les trois chœurs se trouve au livre II, $664 \mathrm{~b}-667 \mathrm{~b}$; voir l'édition commentée de E.B. ENGLAND, The Laws of Plato. Manchester, The University Press, 1921; Vol. I, en particulier les notes de la p. 313 sur l'ivresse et l'initiation religieuse. 
se referme sur des considérations des Mystères qui sont objet de foi, qui sont vérité pour Platon. Les interprétations qui dévalorisent dans le platonisme les sphères du discours autres que celles de la métaphysique spéculative vont en général de pair, d'un point de vue formel, avec une attitude méfiante à l'endroit du discours mythique. Nous verrons plus bas qu'il en est ainsi chez L. Paquet. Ce double dualisme de l'intuition philosophique et de son expression discursive, de la métaphysique et du discours mythique caractérise toute son approche.Mais revenons à l'exposé proprement dit.

Dans la transposition des Mystères, Paquet soutient que Platon a retenu surtout l'aspect visuel, spectaculaire (p. 327). Ni les jeûnes, ni les manipulations, mais l' $\epsilon \pi o \pi \tau \epsilon i a$, les $\delta \epsilon i \varkappa \nu \dot{\nu} \mu \epsilon \nu a$. Le Banquet, le Pbèdre, et la République (VII) sont remplis de ces visions soudaines de l'Idée, identiques à l'époptie des Mystères. Ces rapprochements remontent au livre de Léon Robin, qui mettait en rapport érotique et dialectique, en faisant voir le lien étroit de l'Amour et de la connaissance ${ }^{12}$. Ce qui intéresse ici Paquet, c'est la présence au cour de ce schème analogique de l'union mystique de l'image du regard (p. 333), dans laquelle il retrouve «le mouvement complet du myste se soumettant à l'impératif de la conversion et du regard en direction de l'Objet révélé ». Se tourner, regarder et voir, voilà les trois moments de la philosophie dans Rép. (514a-520c), dans le troisième discours du Pbèdre et dans le discours de Diotime dans le Banquet, où les Mystères sont transposés dans une philosophie de l'ascension dialectique (p. 354). Que ce parallèle existe et qu'il s'agisse plus encore que d'une transposition, d'une véritable intégration de la mystique, nul ne le contestera. Il serait cependant intéressant d'apprendre ce qu'on peut en tirer, en particulier de la prédominance de la VUE, pour arriver à mieux déterminer la nature des objets derniers du platonisme, le Bien, le Beau, l'UN.

Le lien de l'intelligible et du lumineux, explicite dans toute la tradition platonicienne, n'est-il en effet qu'une métaphore? Vision et connaissance, clarté et vérité sont des paradigmes constants du discours philosophique et il n'est pas clair qu'il ne

12. ROBIN, L., La théorie platonicienne de l'amour, Paris, 3e éd., PUF, 1964. 
s'agit là que d'analogies. À cette étape de son enquête, L. Paquet se tourne cependant vers une image susceptible de synthétiser celles du démiurge et du myste et de recevoir les traits des personnages platoniciens, l'amant, le philosophe, le gouvernement : l'image de l'artiste (Chap. XIII).

Comme dans le cas de la démiurgie et des mystères, Paquet voit dans l'attitude de Platon un paradoxe: un rejet de l'art comme copie et une intégration de la théorie de la mimesis. Le chapitre est remarquablement documenté en ce qui a trait aux connaissances techniques de Platon concernant la peinture et les canons de la sculpture. La censure de Platon paraît s'exercer à

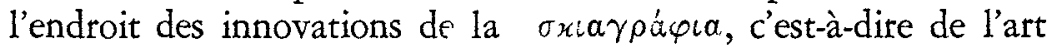
du faux semblant (p. 369) et du simulacre. À vrai dire, la théorie de l'imitation en elle-même (mais comment distinguer l'imitation de l'imitation?) est essentielle à la structure hiérarchique de la philosophie de Platon, où les choses copient les Formes. Paquet montre que la copie artistique n'est pas «copie de la copie», mais bien, à travers la chose représentée, copie du seul Modèle intelligible. Cet obstacle mis de côté, la voie est libre pour l'utilisation de l'analogie de l'artiste en tant que telle. Elle introduit en effet un système de rapports structurés : artiste/œuvre, démiurge/cosmos, artisan/produit, gouvernant/cité, etc..., qui sont tous dans la dépendance de la médiation du regard dans la direction du Modèle (par ex. Rép. 500cd). On notera de manière particulière les rapprochements entre le Législateur et le peintre (500e $\zeta \omega \gamma \rho a ́(\varphi o)$, l'utilisation de la notion d'esquisse, etc... (Lois, $770 \mathrm{~b} \pi \epsilon \rho \imath \gamma \rho a \neq \eta \dot{\eta}$ ). C'est cependant la clarté avec laquelle cette analogie intègre le monde des Formes qui fait son privilège. Puisqu'elle le présuppose à titre de Modèle, elle nous instruit sur les axiomes réels du platonisme: les Formes ne sont en effet, jamais l'objet de démonstrations véritables (p. 389) mais plutôt toujours partie prenante dans des rapports analogiques, susceptibles de nous enseigner la manière dont on les découvre. De cela suit l'importance dans tous ces contextes analogiques de la direction du regard plus encore que de l'homologie modèle/Formes. Dans le débat que nous avons mentionné au début sur la valeur heuristique de l'analogie, L. Paquet semble donc pencher pour une valeur uniquement illustrative. Le reste, la thèse, est un donné apodictique. Ceci limite singulièrement la portée de 
la transposition, comme j'ai déjà commencé à le souligner plus haut. Dans cette perspective, Platon ne transpose que pour illustrer, que pour couler dans un moule, etc... Je crois cette perspective erronée. J'y reviendrai.

Une chose importante intervient ici dans l'exposé : la présentation du Modèle comme «module de rapports», comme ordre, arrangement, proportion. Assurément, tous ces prédicats s'appliquent de manière inégale aux formes intelligibles. Il serait intéressant d'analyser, à la lumière du travail de K. Gaiser et H.-J. Krämer en particulier, ${ }^{13}$ ce qui dans l'analogie du canon artistique facilite pour ainsi dire une intégration du pythagorisme. Ce sujet est effleuré par Paquet (p. 400) et il est certain, on en tombera d'accord avec lui, que la notion de Modèle ne se réduit pas à celle de but mais qu'elle implique, d'une part, une mathématique de la proportion et de la symétrie et, d'autre part, une hiérarchie de niveaux non dualiste (p. 405). L'artisan qui fabrique un objet comme l'artiste qui le représente ont un unique modèle, et leur regard répète la «création » du modèle divin, la forme intelligibile. On ne peut que déplorer à ce stade du travail que L. Paquet n'accorde guère de crédit aux textes fondamentaux du Timée (p. 408), car ces textes représentent le fondement du concept de production seion un Modèle chez Platon. Le mythe n'est pas un «jeu d'esprit» et la figure du Démiurge est plus qu'une figure «mythique ». C'est en effet dans ce texte que le Modèle se trouve expliqué avec netteté, suivant les genres du Sophiste, et qu'il devient possible de saisir clairement ce que le regard regarde quand il voit. L. Paquet s'attache à faire remarquer dans la figure du démiurge les traits du peintre/artiste dégagés plus haut.

Mais qu'en est-il de ces proportions du Modèle (p. 411) ? Ils sont désignés comme une «chaîne de proportions analogues

13. En plus du livre de K. GAISER, Platons ungeschriebene Lebre, Stuttgart, 1963 , dont on pourra consulter toute la première partie sur le rapport des mathématiques et de l'ontologie, il faut mentionner son article, «Platons Menon und die Akademie», Archiv für Gescbicbte der Pbilosopbie, 46 (1964) 241-292, où se trouve étudiée la notion de «schema». Voir aussi le dernier livre de H.J. KRÄMER, Platonismus und bellenistische Philosophie, Berlin, de Gruyter, 1971, pour l'histoire hellénistique de la thèse platonicienne des principes. 
qui descend des Intelligibiles jusqu'au dernier des visibles ». Cette manière de représenter la structure ontologique ne tient pas suffisamment compte de la figure même du Démiurge, qui est ici laissée pour compte, puisqu'on l'interprète abstraitement comme une sorte de mythème du principe de causalité, qu'il est impossible de comprendre hors du mécanisme de l'analogie (p. 414). Cette interprétation est insatisfaisante et écarte sans les discuter d'une part les hypothèses de nature théologique ou cosmologique, tout en reléguant d'autre part le mythe au statut d'un discours illégitime.

Dans un chapitre final qui donne son titre à l'ouvrage, l'auteur rassemble les résultats de chapitres aussi divers (Chap. XIV : La médiation du regard). De quelle médiation s'agit-il? Le regard fait d'abord passer de l'image au Modèle, du simulacre à la Réalité ; il est donc conçu comme mouvement, progression d'un sujet (p. 419) selon de nombreuses étapes, de conversion en retournement, jusqu'à la vue qui est vision. Aux divers niveaux de l'ascension, même aux plus spéculatifs (Sophiste, Parménide) le regard semble privilégié, capable de «discerner le jeu de proportions qui régit une pluralité de données mises en présence ». Le chapitre passe en revue plusieurs textes où le regard est indépendant de ses contextes analogiques d'origine. Les exemples des deux Hippias sont très forcés; ceux de l'Eutyphron et du Ménon le sont moins, mais la formule de L. Paquet est très exagérée : «avant tout le regard qui sait lire le rapport ou la disparité entre l'Idée et les objets qui lui sont comparés. »Car il ne s'agit pas en Ménon (72c) de «lire», mais bien simplement de garder en vue, de considérer à travers toute opacité l'idée. La notion d'un rapport, par laquelle s'introduit subrepticement l'analogie, est absente. L'examen de ces textes sur le «regard de l'esprit». (Rép 618c-e, Pbilèbe 44e.45a \& 65c, Lois $757 \mathrm{bc}$ ) révèle «la hantise singulière d'arracher aux moindres réalités, et a fortiori aux plus imposantes, le secret des systèmes de proportions qui les rapprochent à divers égards.» (p. 437). Par rapport à ces textes, les passages qui font partie de contextes analogiques ont une signification encore plus précise : ils cherchent «avant tout les proportions de la copie au modèle idéal » (p. 441); ce regard est nommé «synoptique» (par ex Lois 965bc). On aura reconnu ici le souci de faire déboucher l'analyse des images platoniciennes 
sur la théorie scolastique de l'analogie; d'un usage de l'analogie désignant les contextes d'origine de certains concepts transposés dans le platonisme, on passe, par l'intermédiaire du regard et de manière non explicite, à une anologie générale des êtres à la réalité fondamentale. Je crois que cette interprétation ne s'applique pas au discours de Platon. En conséquence, tout le monde en conviendra, «c'est une misère que d'avoir la vue faible» (p. 444), texte de l'Hippias qui s'apitoye sur le sort de ceux à qui le regard de l'analogie n'est pas donné.

\section{II}

Où donc travaille-t-on en fixant les yeux sur les Idées?

ARISTOTE, Metaph. (A 9,991a23).

Après avoir exposé le parcours du travail de L. Paquet, il est temps de passer à un examen plus serté de certaines des affirmations qui le structurent. Je ferai d'abord des remarques sur la pertinence des thèses sur l'analogie pour passer ensuite, dans un dernier moment, à une discussion de méthode concernant les rapports de la philologie et de la sémantique.

Un des problèmes majeurs du livre me paraît résulter d'une définition confuse des concepts d'analogie et de transposition, dont l'auteur ne nous instruit jamais en quels points ils se recoupent et quels sont précisément leurs objets. Il se trouve que ces deux concepts sont importés de travaux qui se sont succédé dans l'histoire de l'interprétation ${ }^{14}$. Auguste Diès a d'abord donné une définition approximative de sa notion de «transposition»:

14. DIÈS, A., Autour de Platon, Paris, 1929, 2.volumes. Cf. pp. 16 ss et l'article "La transposition platonicienne», vol. II, pp. 200-449, spécialement p. 444. On doit aussi mentionner les nombreux travaux de Vincenzo CILENTO, dont un recueil d'essais vient d'être publié à Milan. Déjà en 1961 il avait centré autour de ce concept plusieurs de ses recherches dans le recueil Trasposizioni dell'Antico. Saggi su le forme della Grecità al suo 'Tramonto. Milano, R. Ricciardi, 1961. L'introduction propose une utilisation méthodique de la transposition. 
«... il y a au fond de toutes ces théories platoniciennes, à propos desquelles on nous parle si souvent d'emprunts, de survivances, de contaminations orphiques ou autres, une transposition très sûre et très consciente d'elle-même ». Il explique ailleurs qu'il s'agit non seulement de l'ornement littéraire et des métaphores, mais bien de la doctrine. Il n'est nulle part question d'analogie dans le travail de Diès, du moins pas au niveau méthodologique. C'est Paul Grenet, son disciple, qui introduit ce concept pour tenter de pousser plus avant celui de transposition ${ }^{15}$. Il s'agit chez lui, cette fois, de «transposition de l'analogie » religieuse et mythique, mathématique et artistique. Pour lui, Platon reprend à des discours antérieurs la méthode analogique, à la fois comme heuristique et comme illustration du savoir philosophique. L. Paquet situe son travail dans la foulée des deux premiers, mais l'ensemble ne marque pas un progrès dans la définition des concepts de transposition et d'analogie.

Parlons d'abord du concept de «transposition». Je crois ce concept important et utile. Il me paraît judicieux cependant de le distinguer du concept d'analogie en ce qu'il constitue un procédé discursif et textuel, alors que l'analogie est conceptuelle, thématique et abstraite. Cette distinction est grossière et je ne la propose que pour excuser aussitôt le caractère inachevé des remarques que je veux faire sur les termes à distinguer. Dans la pratique de Diès, la transposition était destinée à séparer clairement dans le discours platonicien le bon grain de l'ivraie, c'est-àdire parvenir à réconcilier en les discernant la pureté des intuitions platoniciennes et la grossièreté des éléments discursifs que la philosophie employait pour se définir et/ou s'exprimer. Diès y arrive en montrant que ces éléments ont été transposés, qu'ils sont purifiés par l'Idée plus qu'ils ne la contaminent. Par ailleurs, il fait voir clairement, - et en cela L. Paquet le suit toujours, que ce qui n'a pas été transposé n'en était pas digne. En bref, le pire fut laissé de côté et l'acceptable fut transposé. On peut refuser le principe de cette lecture, on doit néanmoins en respecter les conséquences; il est sûr en effet que dans l'histoire des discours, leur succession et leurs emprunts font spécialement problème dans le cas de discours hétérogènes. Il est certes plus aisé de voir ce qu'Aristote prend à Platon que de mettre au clair

15. GRENET, P., op. cit., p. 16 s. 
ce que celui-ci reprend à l'orphisme. Il est impérieux, que ce soit par le moyen de ce concept de transposition ou par quelque autre, de s'attacher à l'étude des moyens par lesquels une philosophie se construit sur et par la non-philosophie. D'aucuns croient que le langage ordinaire y tient une grande place; à ce niveau du travail sur la langue, à n'en pas douter, ils ont raison. Mais le platonisme a intégré bien davantage, à partir de discours très différents. La transposition des discours n'est pas uniquement le travail du langage.

L: Paquet souligne que la transposition n'est pas «l'utilisation simplement poétique ou littéraire de certaines données de l'expérience, mais bien l'assomption globale de ces données...» (p. 296s). Il faut constater qu'il ne s'agit précisément pas de données, mais de thèses, de propositions, de concepts, que la recherche doit s'attacher à repérer textuellement. L'école de la "Quellengescbichte» a beaucoup fait en ce sens pour raffiner les méthodes; il reste néanmoins beaucoup à faire. Dans cette perspective, il serait utile de réserver le nom d'analogie à ce qui concerne précisément l'expérience, c'est-à-dire à la constitution de rapports inédits pour ce qui n'est pas encore discours identifié et de parler de transposition pour étudier les mécanismes d'emprunts, les transformations, les procédés exégétiques qui ont cours entre les éléments identifiables des discours. Cette étude est très éloignée des procédures de l'analogie et il me paraît essentiel que cette distance soit maintenue. Par là, on convient de distinguer le concept méthodologique de transposition, appliqué à la pratique des textes, du concept métaphysique ou beuristique d'analogie par lequel des rapports sont pensés. C'est ce que ne fait pas L. Paquet; ce qui le requiert dans les textes qu'il étudie n'est jamais clair: procédures analogiques, emprunts textuels, exégèses, changements lexicaux, mais aussi, comme en surplomb, thèses, doctrines, métaphysique. Soucieux par ailleurs d'assurer la grandeur du discours platonicien, il introduit entre philosophie et non-philosophie un fossé qui ne se trouve pas chez Platon. La transposition est marquée dans son livre d'un signe positif qui en dit la valeur; non seulement insiste-t-il sur le «travail de purification auquel doit se livrer le penseur qui prétend construire une authentique analogie philosophique à même les matériaux parfois grossiers de l'expérience humaine concrète» (p. 298); 
mais encore met-il partout l'accent sur cette sorte d'existence séparée du discours philosophique, en tous points semblable à celle de ses objets dans le platonisme. Tout discours ne saurait en être que la copie, l'épure. Il se pourrait néanmoins que cette proposition soit contrainte de se renverser et qu'une étude véritable de la transposition nous enseigne à voir dans le discours philosophique la copie de tout discours.

Il se trouve donc derrière la méthode de l'étude de la transposition une confusion regrettable avec le concept d'analogie, qui prive d'une utilisation positive de la transposition en dénaturant d'emblée la nature des «matières » transposées. Déjà pour Diès, il n'était guère « possible de remuer sans dégoût le terrain de fange d'où Platon transplante la rose pourpre de l'Éros philosophique $\gg$. Après les travaux de Dodds, on sera moins affirmatif sur la rose et plus clair sur la fange ${ }^{16}$. En clair, on acceptera que des contenus et des propositions d'origine identifiable du discours philosophique soient d'abord interrogés littéralement et on ne présupposera pas qu'ils ne peuvent avoir de signification qu'analogique ou transposée dès le point de départ de la lecture.

Ceci me semble en tout cas possible quand il s'agit des contenus étudiés par L. Paquet, en particulier le démiurge et, en général, le regard. S'il se trouve en effet, en deça du regard du Démiurge fixé sur le Modèle, quelqu'autre regard dont le premier n'est que le support analogique transposé, alors il doit pouvoir s'en séparer. Or tel n'est pas le cas; hors du support du démiurge, il n'existe que la fiction des interprètes : le principe de causalité. Cette fiction ne correspond pas à la philosophie platonicienne, et celle-ci n'est pas primitive pour autant. Il faut en dire davantage, à la suite de Beierwaltes, de la lumière, du regard, etc... : il n'y a rien derrière ces éléments et ils ne sont pas des images ${ }^{17}$. Il ne

16. DIÈS, A., op. cit., p. 432.

17. Voir W. BEIERWALTES, Lux Intelligibilis. Untersuchung zur Lichtmetaphysik der Griechen. München, 1957; Beierwaltes a donné un exemple de cette pratique de l'interprétation dans un magnifique article sur Plotin, Die Metaphysik des Lichtes in der philosophie Plotins, Zeitschrift für philosopbische Forscbung 15 (1961) 334-362. On consultera également Hans BLUMENBERG, Wahrheit, Licht und Erkenntnis in der griechischen Philosophie bis Demokrit. Ein Beitrag zur Erforschung des Zusammenhangs von Sprache und philosophischen Denken. Archiv für Begriffsgescbichte X (1966), avec le compte rendu critique de W. Beierwaltes, Göttingische Gelebrte Anzeigen (1968) 1-13. 
faut donc pas étendre indûment l'usage de l'analogie et en tirer une pratique de l'histoire de la philosophie qu'on pourrait qualifier de fictive, en particulier quand il ne s'agit pas de thèses mais de procédures. Nos remarques veulent en effet faire voir que la procédure de transposition d'un texte ou d'un contexte dans une philosophie ne suffit pas à constituer une thèse par analogie. Cette dernière n'est toujours que la conceptualisation, à partir d'un rapport, d'entités métaphysiques inédites. Leur caractéristique est d'être absolument indépendantes du rapport par lesquelles elles ont été conçues et on peut dire que dans plusieurs cas, ce rapport ne sert qu'à les illustrer. L'analogie constitue thèses et concepts, la transposition les reprend dans un discours différent, telles quelles, de telle façon que le transposé n'est jamais indépendant de son origine.

Il me semble important enfin, à ce chapitre de l'analogie, de faire une remarque. Peter Geach a distingué les analogies occasionnelles et les analogies systématiques ${ }^{18}$. Les unes concernent le rapport de termes particuliers, les autres de systèmes entiers de descriptions. En ce qui concerne le regard du monde intelligible, il n'est pas clair s'il s'agit d'une analogie occasionnelle ou systématique. Cela vient en particulier d'une question de méthode, où sont mêlées l'analyse lexicale et la transposition de contextes entiers. L'analogie du regard en effet n'opère-t-elle que dans le cas de $\beta \lambda \lambda^{\prime} \pi \epsilon \imath$, , ou en est-il ainsi également des verbes dont Paquet ne dit rien: $\delta \rho \bar{a} \nu, \vartheta \in \tilde{a} \tau \vartheta a^{\prime}, \quad \vartheta \varepsilon \nu \rho \leqslant \tilde{l} \nu$ etc...? Une analyse de nos huit exemples, que j'ai extraits du livre pour leur représentativité, nous montrerait que plusieurs emplois sont souvent indifférents. Je crois donc qu'il y aurait avantage à étudier non seulement l'ensemble du champ sémantique du regard, mais encore tout le système des contextes qui sont avec lui transposés. Autrement on n'a affaire qu'à des parallèles lexicaux, intéressants d'un point de vue philologique, mais sans grande portée philosophique. En effet on ne peut rejoindre au niveau lexical que des analogies tout à fait occasionnelles. En suivant Peter Geach, on accordera que l'analyse d'un terme particulier peut être suggestive ou même son emploi métaphorique inévitable, mais ceci ne nous instruit pas sur l'usage transposé

18. GEACH, P., Mental Acts. London, 1957 ; pp. '75ss. Il s'agit ici de l'analogie susceptible de faire connaître les actes mentaux. 
d'un autre terme de la même famille. Si l'on dit que «regarder ( $\beta \lambda \epsilon \epsilon \epsilon \iota \nu$ ) les idées » est clair, cela ne nous dit pas en quel sens on devrait comprendre, par exemple, «regarder (ópã $\nu$ les idées ».

Il faut, pour terminer ce développement, insister sur une ligne de direction importante du travail de L. Paquet. Puisqu'il privilégie dans l'analogie l'activité plutôt que les termes, c'est-àdire qu'il établit des rapports entre divers termes et l'activité qui y préside, il n'échappera à personne que cette recherche est importante du point de vue des «personnages» du platonisme. On parlerait ici volontiers, d'un point de vue sémiotique, d'une typologie des emplois et des rôles, partie importante dans l'élaboration d'un modèle actantiel du discours platonicien. L'artisan, l'ouvrier, le démiurge, le myste, l'artiste, le roi, le philosophe, le législateur, l'amant sont les principaux rôles sur cette scène: le travail de Paquet nous conduit à remarquer comment chacun dans son activité propre est contraint de $《$ regarder $»^{19}$. Mais qui donc est le premier sujet dont les autres ne sont que des analogués? On se rendrait compte, je crois, que c'est le philosophe. Il est piquant d'avoir à constater, après l'avoir souligné au niveau des discours, qu'encore au niveau des rôles le philosophe est présenté comme le sujet regardant où sont transposés tous les autres rôles sociaux, dans leur pureté. Encore ici cependant, on sera tenté de renverser ce mouvement de l'analogie et de considérer le philosophe, autant au niveau de la production qu'au niveau de ce qu'il voit, comme le rôle dernier, le personnage de la reprise, la transposition de la chouette. Il faut savoir gré à Paquet d'avoir, en choisissant ce terme du regard, décentré quelque peu le foyer du discours platonicien et de l'avoir porté sur les fonctions et les rôles plutôt que sur les Idées. Ce déplacement est instructif, car ce que sont l'Idée et le Modèle sans le regard

19. Il me paraît utile de mentionner ici un travail qui n'est jamais cité par les historiens de la philosophie; son obscurité est certes légendaire, même pour les spécialistes de la rhétorique dont il émane, mais il s'agit d'un ouvrage qui a des affinités certaines avec le travail de la sémiologie et, en particulier, avec la recherche de structures narratives dans d'autres discours que les récits. Il s'agit de Kenneth BURKE, A Grammar of Motives. Berkeley, University of California, 1969 (1ère édition, 1945). Son étude du discours philosophique (pp. 127-320) utilise les catégories suivantes: scène, agent en général, acte, action et but. 
porté sur elles, il est difficile de le savoir; mais ce qu'est le regard sans les sujets qui regardent, nous le savons : il n'est rien. Il y a donc ici un contenu irréductible, intransposable du platonisme : il s'agit des personnages. Il est notoire que celui du philosophe veut transposer tous les autres; cette velléité correspond à la pente naturelle de l'analogie. Ce que le peintre est à l'esquisse, le philosophe l'est à la législation, et ainsi de suite. Le bénéfice d'une recherche sur l'activité et les rôles et, en particulier, sur le regard est de nous montrer que le rôle de philosophe s'évanouit si on le prive des prédicats repris de tous les autres rôles. Il ne reste plus de verbes, mais que l'adverbe «eminenter» (xvpíws ). ${ }^{20}$

J'en viens finalement aux questions qui me sollicitent le plus dans ce livre. Elles concernent le rapport de la philologie, comme discipline des textes, à la sémantique, comme théorie linguistique. Il est notoire que nous nous trouvons ici en face d'un renversement de situation tout à fait intéressant : en effet, après avoir fourni autant les exemples que la théorie de l'étude des textes, - et ce en particulier dans la philologie allemande du début du siècle, - la philologie se trouve en situation de recevoir de la linguistique ses consignes et ses méthodes. C'est en Allemagne que ce problème est le plus débattu actuellement, et on m'excusera d'avoir à faire référence si souvent à des débats auxquels nous aurions tort de ne pas nous intéresser. Une chose est certaine : il est désormais impossible de ne pas tenir compte dans les recherches philologiques des procédures de la sémantique et en particulier de l'état des recherches sur l'analyse des discours.

$\hat{A}$ ce chapitre, le livre de L. Paquet est contemporain des Leçons sur le langage de Max Müller. Il ne tient compte en effet ni des champs sémantiques, ni de l'analyse syntaxique, ni de la rhétorique. Pour le mieux faire voir, je retiendrai de son travail trois problèmes de méthode reliés à des questions d'ordre sémantique: d'abord brièvement la définition de la signification, ensuite le problème des «images» et des métaphores et enfin la question de l'étymologisme.

20. Sur l'analogie, voir pour Platon, outre le livre déjà cité de R. Robinson, celui de D.B. BURRELL, Analogy and pbilosopbical Language, Yale University Press, 1973. Il faut aussi citer, M. MacDONALD, «The philosophical use of Analogy 》, dans Logic and Language. Éd. A. FLEW, New-York, 1965 (Anchor Books), pp. 80-100, et D. EMMETT. The Nature of Metapbysical Tbinking, London, 1949. 
Il n'est que de relever d'abord le vocabulaire de sa démarche pour saisir le degré de précision de la notion de signification chez L. Paquet. On y parle beaucoup «d'aire notionnelle», des «attaches sensibles» d'un terme, de sa «charge», et surtout de ses 《nuances», qui sont «senties», «perçues», « intuitionnées», «vécues». Je résumerai mon propre sentiment en disant que nous avons affaire à une analyse intuitive. J'en illustrerai la difficulté de la manière suivante: L. Paquet s'étonne de ce que

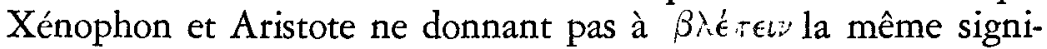
fication que les auteurs littéraires dont il a fait largement l'analyse. Cette constatation est de sens commun, car elle concerne la diversité des objets des discours. Il ne découle pas de cette constatation que la signification du terme diffère: "regarder » y veut toujours dire «regarder», mais, au lieu de Zeus ou d'Alceste, on regarde devant soi ou encore un problème. $\beta \lambda \epsilon ́ \pi \epsilon \iota \nu$ admet un grand régime de compléments, tout comme voir en français, et il n'y a pas, entre " voir une chose ou une personne » et «voir un problème», une différence de «nuance» mais une différence de contexte: la signification, pour autant qu'on la puisse définir ainsi, ne change pas, pour la bonne raison qu'elle n'appartient pas à voir, mais à toute la phrase. Ceci est assez clair en linguistique depuis longtemps. Il y a donc quelque difficulté à lire toutes ces pages appliquées à retrouver nuances et significations dans autant de textes qu'il s'en trouve pour avoir de significations différentes. Il faut laisser de côté en philologie ce lexicalisme ruineux et inutile et adopter des méthodes plus précises. Je noterai en passant, une raison souvent mentionnée pour la persistance en philologie de cette attitude vis-à-vis des lexèmes isolés. Cette méthode a été nourrie pendant longtemps par un double besoin : premièrement l'activité lexicographique ; on peut dire sans ambages que les dictionnaires des langues anciennes comptent parmi les plus parfaits qui soient, les langues étant mortes. Ce sont des dictionnaires de textes. Secondement, la recherche des sources, dont la grille de mots est l'outil le plus commun; c'est ainsi que toute une période n'a connu que des enquêtes diachroniques où un mot est étudié chez une brochette d'auteurs, aux fins de déterminer l'originalité des uns et des autres, mais aussi de repérer à travers lui les états d'évolution d'une doctrine. 
Dans le cas du verbe $\beta \lambda \epsilon ́ \pi \epsilon \iota \nu$, le choix qu'on en fait peut être double: ou bien on étudie ce mot pour voir les contextes qu'il admet, ou bien on étudie tous les mots qui comme lui sont admis dans tel et tel contexte. L. Paquet ne fait ni l'une ni l'autre de ces démarches. S'il se rapproche de la première il n'étudie pas systématiquement les divers contextes admis par le verbe, autant littéraires que philosophiques. Ce type d'analyse requiert une considération détaillée de la syntaxe, c'est-à-dire des constructions précises où le verbe se trouve pris (compléments, prépositions, etc...) ${ }^{21}$. Paquet n'a retenu que les prépositions et il s'étend parfois à discuter les compléments. Par ailleurs, aucun des autres verbes susceptibles d'exprimer l'idée de regard ou d'être insérés dans des contextes de cette nature n'est vraiment examiné. En particulier, les exemples de Timée (47bc) et de Lois (904b) confirment l'emploi du verbe $\dot{o} \rho \tilde{a} \nu$ dans des contextes philosophiques, avec l'intelligible pour complément.

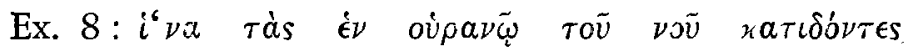

$$
\begin{aligned}
& \pi \epsilon \rho \iota b \delta o v s \quad \chi \rho \eta \sigma a i, u \epsilon \vartheta a \text { é } \pi \iota \text { tàs } \pi \epsilon \rho \iota p o \rho \dot{s} s
\end{aligned}
$$

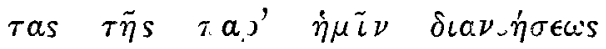

Ayant contemplé les mouvements périodiques de l'Intelligence dans le Ciel, nous les utiliserons, en les transportant aux mouvements de notre propre pensée, [lesquels sont de même nature, mais troublés, alors que les mouvements célestes ne connaissent pas de troubles.]

\section{Timée 47b6-7 [Trad. A. Rivaud]}

21. Jean DUBOIS, dans son travail sur Le vocabulaire social et politique en France, 1880-1890, Paris, 1958, donne les consignes générales suivantes du travail lexicographique: définir le champ lexical et prendre position par rapport à l'idée de système; se situer par rapport à la synchronie et à la diachronie; déterniner le moment historique et inventorier les sources et les documents qui sont synchroniques. Ensuite, faire l'enquête de la manière suivante: an sujet de toute unité soumise à l'analyse, demander 1. la signification du singulier et du pluriel; 2 . les valeurs d'emploi opposées; 3 . le rapport entre le nombre du mot et les qualificatifs; 4. les substituts et les variantes; 5 . antonymes ; 6. zone d'appartenance du discours : lexique moral, politique, etc ..; 7. niveaux de langue: usuel, technique; 8. système des préfixes et des suffixes; 9 . procédés de dérivation et de composition; 10 . étude des unités sémantiques complexes : expressions dont le sens varie tout en demeurant quantitativement constantes (lexies). 
Cet exemple est clair, puisque discutant les raisons de la vision chez l'homme, il ne distingue pas la vision des mouvements du ciel de celle de la pensée, toutes deux introduites par óoẫ L'exemple des Lois est du même registre. Les considérations de méthode (pp. 66-70) confinent donc au vœu de la rigueur plutôt qu'aux conditions de son exercice.

Cela dit, je m'en voudrais de laisser entendre que la philologie n'a qu'à se tourner vers la linguistique pour apprendre comment procéder. Il s'en faut que la sémantique soit elle-même correctement constituée; la théorie des champs a été bien critiquée et il faut tenir compte de ces critiques. On peut et on doit néanmoins en principe retenir que le lexique d'un texte laisse percevoir des systèmes d'oppositions. Sans doute variables et non exhaustives, ces oppositions arrivent à mieux structurer l'analyse de vocables que la seule considération de termes isolés. On peut en voir l'exemple dans plusieurs travaux, et principalement dans l'étude de John Lyons sur Platon ${ }^{22}$. Celui-ci définit la signification en termes de relations antérieures: incompatibilité, antonymie, hyponymie et il représente ces relations comme des relations sémantiquement primaires, exprimables selon la négation, l'implication et l'équivalence. On s'intéressera surtout à l'application très stimulante de ses principes de sémantique structurale aux vocables philosophiques de Platon ( $\left.\dot{\epsilon} \pi \iota \sigma \tau \dot{\eta} \mu \eta, \tau \dot{\epsilon} \chi \nu_{i}\right)$. Je crois qu'il s'agit là d'une philologie renouvelée qui peut contribuer de manière originale à l'histoire de la philosophie, en plus de fournir à la théorie linguistique une certaine épreuve de ses hypothèses.

22. Voir, outre les travaux généraux de Jost Trier, ceux de A. JOLLES, «Antike Bedeutungsfelder. Beitrage zur Geschichte der deutschen Sprache und Literatur 》, PBB 58 (1934) 97-109, accessible facilement dans le recueil Wortfeldsforschung. Darmstadt, Wissentschaftliche Buchgesellschaft, 1973; pp. 104-115. Pour une analyse complète d'un lexème, cf. L. GRAZ, Le feu dans l'Iliade et l'Odyssée. NYP, champ d'emploi et signification, Paris, Klincksieck, 1965 (Etudes et commentaires), avec les remarques de Y.M. Charue, «Notes de sémantique homérique. Constitution du champ sémantique et analyse des unités», Recherches en pbilologie et en linguistique (1968) 95-108. Voir aussi C. DE HEER, Má $\chi \alpha \rho-\epsilon \dot{v} \delta a i \mu \omega \nu-b ̋ \lambda \beta$ os $\epsilon i \tau v \chi \dot{\eta}$ s A Study of the semantic field denoting happiness in ancient Greek to the End of the Fifth Century. Amsterdam, Hakkert, 1969, qui utilise les principes de la sémantique de Ullman. Enfin, John LYONS, Structural Semantics. An analysis of part of the Vocabulary of Plato. Oxford, Blackwells, 1963 (Publications of the Philological Society, XX). 
Je passe maintenant au fait que L. Paquet considère la famille verbale à l'étude comme une image (p. 70) et souvent comme une métaphore. L'existence présumée d'un registre d'application littérale du verbe, c'est-à-dire le regard physique, concret gauchit notoirement l'ensemble des analyses. Outre le fait que le passage de significations concrètes à des significations plus abstraites constitue une thèse depuis longtemps périmée, - puisqu'elle est historiquement indémontrable, - elle véhicule une définition incorrecte de la métaphore. Cette définition suppose un en-deça de l'expression métaphorique lexicale, un au-delà qui se révèle toujours inexistant. La métaphore se présente ici au niveau lexical comme l'équivalent de la transposition: la métaphore supplée à l'expression de l'abstrait les moyens qui lui manquent pour se dire. Pas plus que «comprendre » n'est une métaphore parce qu'on peut y voir une sorte de "prendre ensemble» d'abord concret, regarder n'est pas une image. Il n'y a pas d'en deça, pas de substitut concret ou abstrait qui en serait la littéralité. Le regard des idées est un regard. Cette proposition n'est pas équivoque. Ceci n'est pas le cas, par exemple du «voile de la Nature » que la science tente de soulever (Héraclite) ou encore du « cours de la Nature ${ }^{23}$.

Les métaphores et les images sont des objets fuyants, qui se laissent mieux manipuler si on les considère lorsqu'elles forment système que lorsqu'on tente de les considérer isolément. Seul un syntagme peut tetre métaphorique relativement à ses composants et encore à ce niveau les métaphores ont-elles tendance à se lexicaliser rapidement. Dans le cas du discours philosophique, il est intéressant de les étudier selon des ensembles tels que celui de la lumière, etc... L'oeil de l'âme, la clarté des idées, le soleil du Bien s'analysent selon des rapports précis ; mais même intégré dans ce système, de quoi le regard est-il l'image ou la métaphore? C'est cela qu'il conviendrait ici de se demander. Il convient également d'accorder à ces questions toute l'importance que leur donne Hans Blumenberg dans son travail sur la

23. Voir H. GALINSKY, «Cursus Naturae. Der Weg einer antiken kosmologischen Metapher von der alten in die neue Welt. Ein Beitrag zu einer historischen Metaphorik der Weltliteratur », Arcadia I (1966), 277-311. 
《métaphorologie ${ }^{24}$. La philologie a donc ici à reprendre à la rhétorique ce qu'elle peut en tirer pour se rigoriser. Depuis Eucken, beaucoup de chemin a été parcouru.

Dans l'histoire des concepts, la considération des vocables a toujours tenu une place d'importance. L'ajout d'un déterminant suffit en général à transformer n'importe lequel des mots du vocabulaire en «concept». Cette inflation me paraît à l'œuvre dans la notion du «concept de $\beta \lambda \epsilon ́ \pi \epsilon \iota \nu \gg$ Cette inflation se double d'un étymologisme inutile; non qu'il s'agisse de la recherche de l'etumon, du sens originel du signifiant, mais d'une remontée temporelle vers les origines des significations transposées. Dans un livre assez récent pour qu'on le cite comme une pièce nouvelle à ce dossier, F. Kainz a voulu soutenir que l'étymologie philosophique est non pas linguistique mais «sémantique» ${ }^{25}$. Il ne s'agit plus de rechercher, comme l'a dénoncé Buyssens, le rapport intrinsèque entre un signifiant et son signifié, mais néanmoins d'établir une chaîne des signifiés jusqu'aux signifiés premiers, originaires, véritables bases des concepts. Suivant Buyssens donc pour la question de l'étymologie, Kainz s'en sépare sur la question de l'élaboration d'une sémantique; en effet pour Buyssens, la sémantique est linguistique et se limite aux signifiants. Pour Kainz, la sémantique philosophique intègre les signifiés. Il étudie par exemple les processus de différenciation des catégories axiologiques: roi $\iota_{\iota s}$, d'abord séparation, tri, puis jugement, discernement. L'entreprise de L. Paquet réunit ce double motif

24. Hans BLUMENBERG, «Paradigmen zu einer Metaphorologie», Archiv für Begriffsgeschicbte. VI (1960). Rudolf Eucken a consacré un mémoire à ce sujet, Über Bilder und Gleichnisse in der Philosopbie, Leipzig, 1880, en plus de sa Gescbicbte der pbilosopbiscben Terminologie, Hildesheim, Olms, 1964 (1879). Voir les développements sur Platon, p. 16s, où il donne une liste des termes dont Platon a consacré l'usage technique et philosophique. Voir aussi W.K. Wimsatt, Philosopbic Words, New Haven, 1948. Dans leur bibliographie du langage métaphorique dans l'Antiquité, Pöschl, Gartner et Heyke ont accordé une grande place à Platon, mais le terme ne fait l'objet d'aucune référence. Voir, pour Platon, pp. 303-320, dans Bibliograpbie zur Antiken Bildersprache, Heidelberg, Carl Winter, 1964 (Heidelberger Akademie der Wissenschaften). Cette bibliographie est indispensable pour l'étude des figures du discours dans l'Antiquité.

25. KAINZ, F., Pbilosopbische Etymologie und bistorische Semantik, Wien, 1969 (Öester. Akad. der Wiss. Phil.-hist. Klasse, Sitzungsber. 262 Band, 4. Abh.) 
de l'hypostasiation d'un terme en concept et d'un étymologisme criticable. Il suffit de citer à la suite la somme de nos exemples pour voir qu'il est impossible de trouver chez Homère un sens originaire dont le sens platonicien serait un développement. $\bar{A}$ cette thèse, Liddell-Scott-Jones apportent une confirmation. Pour

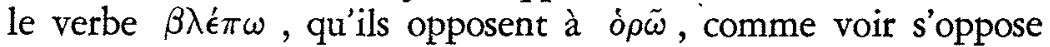
à percevoir-être conscient, ils citent autant d'emplois anciens que classiques dans des contextes concrets et abstraits. Il n'y a donc pas de concept originaire du regard, mais des discours porteurs de significations diverses où ce verbe figure. Que je sache, Platon n'a pas fait du regard un «concept » déterminant et technique de sa philosophie, dont il y aurait lieu de confronter les définitions et les applications. Il s'agit plutôt d'un fait de discours, comme mille autres, dont le privilège est de se déplacer entre des concepts qui eux sont techniques et définis. De tels déplacements peuvent faire l'objet d'études littéraires et linguistiques, mais il y a quelque difficulté à les traiter «conceptuellement ».

Il faut donc revenir à ce que nous disions plus haut et faire le vœu d'une philologie pour laquelle les changements sémantiques seront d'abord des modifications de contextes : réduction, expansion, altération. Opérant de manière rigoureuse sur des textes et sur des langues «finies», cette philologie pourra cette fois contribuer aux progrès de la sémantique linguistique, toujours en quête d'exemples et de démonstrations précises.

J'arrête ici ces quelques remarques critiques. Mon désaccord avec la méthode du livre est profond. Je dois cependant dire mon admiration pour la qualité de sa facture. Les exigences de la philologie sont considérables; en plus d'une connaissance des textes et des langues, celui qui veut la pratiquer doit toujours retraverser, comme allant contre un vent adverse, l'épaisseur toujours plus grande des commentaires qui s'interposent entre le texte et lui. Il se produit une sorte de calcification du discours philologique, toujours plus lourd, plus chargé d'appareils complexes, de systèmes de renvois dont on est en droit de se demander s'ils trouveront un jour leur limite. Le télescopage des sources, la terrible Quellengeschichte, sont souvent des obstacles massifs à une analyse nette des discours. Si on peut regretter chez L. Paquet certaines notes (p. 234, p. 67) qui n'ont que peu de rapport avec 
la progression de l'étude, on s'accordera à reconnaitre dans son livre un travail articulé et magnifiquement documenté. Une bibliographie par rubriques, des index faciliteront la lecture de cet ouvrage stimulant dont il faut être reconnaissant au Conseil des Arts d'avoir facilité la publication. Il n'est pas si fréquent qu'une philologie d'aussi bonne qualité trouve chez nous à se rendre publique, et l'on mesurera l'estime que je porte à ce livre à la longueur des remarques critiques qu'il a provoquées chez moi et dont je veux en terminant m'excuser.

Université du Québec à Montréal 